\title{
Adaptação dos Testes de Lactato Mínimo, Potência Crítica e Limiar Anaeróbio para Avaliação da Transição Anaeróbia-Anaeróbia em Protocolo Específico para o Tênis de Mesa
}

Assessment of the Aerobic/Anaerobic Transition in a Protocol Specific for Table Tennis

Érika Alves Morel'

Alessandro Moura Zagatto

1. Laboratório de Pesquisa em Fisiologia do Exercício - Lapefe - Universidade Federal de Mato Grosso do Sul - UFMS, Campo Grande, MS.

Endereço para correspondência: Alessandro Moura Zagatto, UFMS - Departamento de Educação Física. Av. Costa e Silva, s/n, Cidade Universitária - 79070-900 - Campo Grande, MS. Caixa Postal 549. E-mail: azagatto@yahoo.com.br

Submetido em 26/11/2007 Versão final recebida em 06/05/2008 Aceito em 05/07/2008

\section{RESUMO}

Os objetivos do estudo foram verificar a adaptação dos testes de lactato mínimo, freqüência crítica e limiar anaeróbio em protocolo específico para o tênis de mesa para avaliação da transição anaeróbia-anaeróbia; verificar a reprodutibilidade do teste de lactato mínimo; e verificar a associação desses procedimentos específicos com o limiar anaeróbio determinado em corrida em esteira rolante $\left(L A n_{\text {est }}\right)$. Para isso, foram estudados 11 mesatenistas com tempo mínimo de treinamento de dois anos. Foram aplicados os testes de: 1) lactato mínimo em duas ocasiões para análise da reprodutibilidade ( $\left(\mathrm{AC} \mathrm{Min}_{1}\right.$ e LACmin 2$)$; 2) teste de freqüência crítica (fcrit); 3) teste incremental para determinação do limiar anaeróbio através da concentração fixa de lactato de $3,5 \mathrm{mM}\left(\mathrm{LAn}_{\text {esp } 3.5}\right)$ e regressão linear bissegmentada $\left(L A n_{B}\right)$, todos em procedimentos específicos para o tênis de mesa; e 4) o limiar anaeróbio em corrida aplicado em esteira rolante $\left(L A n_{\text {est }}\right)$ determinado pela concentração fixa de lactato de $3,5 \mathrm{mM}$. Nos procedimentos específicos foi utilizado um lançador de bolas mecânico como ergômetro e os participantes realizaram golpes apenas de forehand. Como procedimentos estatísticos foram utilizados os testes de Bland-Altman, correlação produto-momento e ANOVA one way, com nível de significância de 5\%. As intensidades de LACmin (59,40 $\pm 4,06$ e

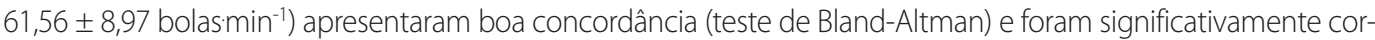

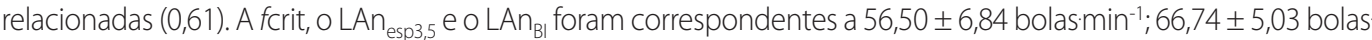
min $^{-1} ;$ e 62,67 $\pm 7,25$ bolas min $^{-1}$, respectivamente. Foi verificada apenas diferença significativa entre as intensidades de $L A n_{\text {esp } 3,5}$ e de fcrit. Foram encontradas correlações significativas entre $L A C \min _{1}$ e LACmin $2(0,61)$, LACmin 1 e fcrit $(0,61), L A C \operatorname{Lin}_{2}$ e fcrit $(0,69)$, $L A n_{\text {esp } 3,5}$ e $L A n_{B 1}(0,70)$, enquanto que o $L A n_{\text {est }}\left(9,11 \pm 1,94 \mathrm{~km}^{-1} \mathrm{~h}^{-1}\right)$ foi apenas correlacionado com a intensidade de fcrit $(0,73)$. Desse modo, pode-se concluir que o teste de LACmin específico é reprodutível e que esse procedimento, assim como o teste de fcrit, pode ser aplicado para avaliar a transição aeróbia-anaeróbia no tênis de mesa e que o LAn ${ }_{\text {est }}$ deve ser utilizado com cautela na avaliação de mesatenistas.

Palavras-chave: lactato, lançador de bolas mecânico, reprodutibilidade, especificidade, tênis de mesa.

\section{ABSTRACT}

The aims of the present study were to adapt the lactate minimum, critical power model and anaerobic threshold tests for assessment of the aerobic-anaerobic transition in specific test for table tennis; to verify the reproducibility of the lactate minimum test and to verify the relationship between these specific procedures with the anaerobic threshold determined in running on treadmill $\left(A n T_{T R}\right.$ ). Eleven male table tennis players participated in the study. The participants performed four tests (three specific tests and a conventional test performed on a treadmill): 1) lactate minimum test (LACmin) (applied twice for reproducibility analyses - LACmim1 and LACmin2); 2) critical frequency test (critf); 3) incremental test for anaerobic threshold determination by 3.5 $\mathrm{mM}$ steady lactate concentration $\left(\mathrm{AnT}_{3.5}\right)$ and bi-segmented linear regression $\left(\mathrm{AnT}_{\mathrm{B}}\right)$ methods, all in specific protocol; and 4) anaerobic threshold on running ( $\mathrm{AnT}_{\mathrm{TR}}$ ). Specific tests were applied by using a mechanical ball thrower to control the intensity of the exercise. The Bland-Altman plot, product-moment correlation and One-way ANOVA tests were used as statistical procedure, with significance level at 5\%. The LACmin intensities

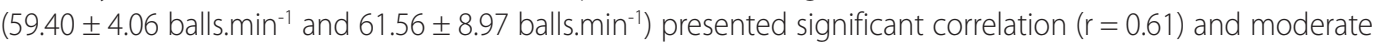
concordance verified by Bland-Altman plot. The critf, $A_{n T} T_{35}$ and $A_{n} T_{B 1}$ intensities corresponded to $56.50 \pm 6.84$

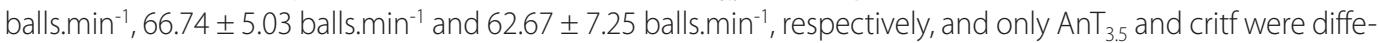
rent. Significant correlations were obtained amongst LACmin ${ }_{1}$ and critf $(r=0.61), \mathrm{LACmin}_{2}$ and critf $(r=0.69)$, and $A n T_{35}$ and $A_{n T} T_{B 1}(r=0.70)$. Anaerobic threshold determined in running $\left(9.11 \pm 1.94 \mathrm{Km} . \mathrm{h}^{-1}\right)$ was only correlated with the critf $(r=0.73)$. Thus, in conclusion, the lactate minimum test specific for table tennis is a reproducible procedure, as well as the critf test can be applied for evaluating aerobic-anaerobic transition. Neverhteless, the anaerobic threshold applied in running on a treadmill must be used with care in table tennis.

Keywords: lactate, mechanical balls thrower, reproducibility, specificity, table tennis. 


\section{INTRODUÇÃO}

A avaliação do componente aeróbio, principalmente em atletas é importante para verificar o estado de treinamento e também para a prescrição da intensidade de esforço, sendo a intensidade correspondente à transição aeróbia-anaeróbia a mais utilizada para tal. Dentre os processos que mensuram o ponto de transição aeróbia-anaeróbia, os testes de máxima fase estável de lactato, limiar anaeróbio individual, limiar anaeróbio através de contração fixa (3,5e 4,0mM) e lactato mínimo são os procedimentos invasivos mais utilizados, enquanto que o teste de potência e/ou velocidade crítica é o procedimento não-invasivo mais empregado. Porém, esses procedimentos são geralmente aplicados em eventos esportivos ou ergômetros não específicos para o esporte como a corrida, o cicloergômetro, a natação e outros; a adaptação desses procedimentos para condições especificas para os esportes como tênis, basquete, vôlei e outros são menos investigados. Como existem poucos estudos aplicando esses procedimentos de avaliação em condição específica para o esporte, os atletas dessas modalidades esportivas são geralmente avaliados em ergômetros convencionais, como corrida em esteira rolante ou pista e também em cicloergômetro.

Em esportes com raquetes existem poucos trabalhos que aplicaram esses procedimentos para avaliar a transição em teste específico e, dentre os estudos existentes, a grande maioria foi aplicada principalmente no tênis ${ }^{(1,2)}$, badminton ${ }^{(3,4)}$ e squash ${ }^{(5)}$. A necessidade de avaliação específica em esporte já é relatada em muitos trabalhos na literatura, inclusive em estudos em esportes com raquetes $s^{(2,3,5,6)}$. Mas no tênis de mesa, a aplicação de procedimentos específicos ainda é escassa $^{(7-10)}$. Diante dessas informações, surge a necessidade da elaboração de procedimentos de avaliação que respeitem as características específicas do tênis de mesa, auxiliando na avaliação aeróbia específica dessa modalidade esportiva, assim como em sua prescrição e monitoramento de intensidade de treinamento. Para isso, é necessário encontrar uma ferramenta que seja confiável e que torne possível essa aplicação.

Desse modo, os objetivos deste estudo foram: 1) aplicar os testes de lactato mínimo, potência crítica e limiar anaeróbio adaptados para testes específicos para o tênis de mesa; 2 ) verificar a reprodutibilidade do teste de lactato mínimo nesse esporte; e 3) verificar a associação entre esses testes específicos com o limiar anaeróbio determinado em corrida em esteira rolante.

\section{METODOLOGIA}

Foram participantes deste estudo 11 mesatenistas das cidades de Campo Grande/MS e Rio Claro/SP (nove homens e duas mulheres), todos filiados à Confederação Brasileira de Tênis de Mesa (CBTM) (idade $=18,7 \pm 7,13$ anos; massa corporal $=61,28 \pm 5,35 \mathrm{~kg}$; estatura $=1,76$ $\pm 0,06 \mathrm{~m}$; e percentual de gordura $=14,7 \pm 7,11 \%$ ) e tempo mínimo de treinamento de dois anos. Esses atletas participaram do estudo de modo voluntário e assinaram um termo de consentimento livre e esclarecido, que foi aprovado pelo Comitê de Ética em Pesquisa da Universidade Federal de Mato Grosso do Sul.

\section{PROCEDIMENTOS EXPERIMENTAIS}

Os testes foram aplicados em um período de duas semanas com intervalo mínimo de 24 horas entre cada avaliação. Os participantes foram informados a não realizar exercícios extenuantes externos ao estudo. Sempre antes de cada teste específico, os atletas realizaram alongamento livre e aquecimento na mesa com auxílio de um robô lançador de bolas (Robo Pro Plus, Tibhar, Alemanha). O aquecimento teve a duração de cinco minutos e a freqüência de disparo de bolas do robô foi ajustada em aproximadamente 41 bolas min ${ }^{-1}$. Todas as regulagens do robô durante o aquecimento foram iguais às aplicadas nos testes e serão mencionadas posteriormente. Os testes de mesa iniciaram-se cinco minutos após o aquecimento. $\mathrm{O}$ aquecimento no teste em esteira teve duração de cinco minutos e intensidade correspondente a $6 \mathrm{~km} \cdot \mathrm{h}^{-1} \mathrm{com}$ inclinação de $1 \%$. Em todos os procedimentos os participantes foram encorajados verbalmente a realizar esforço máximo.

\section{TESTES ESPECÍFICOS PARA O TÊNIS DE MESA}

Em todos os testes específicos os mesatenistas foram submetidos a simulações do jogo de tênis de mesa realizando apenas ataques de forehand em bolas disparadas por um robô lançador de bolas de tênis de mesa (Robo Pro Plus, Tibhar, Alemanha). O lançador de bolas possui ajustes de velocidade da bola, oscilação lateral da bola nos lançamentos e também freqüência de disparo de bola. Tanto a velocidade quanto a oscilação lateral foram mantidas constantes em todos os testes específicos e ajustadas conforme especificação do equipamento (velocidade correspondente à unidade 4 e oscilação lateral correspondente a unidade 5, conforme escala de especificação do equipamento). A velocidade da bola na escala 4 correspondeu a velocidade de aproximadamente $35 \mathrm{~km} \cdot \mathrm{h}^{-1}$ mensurada por um radar (StalkerPro, Stalker, EUA) e a oscilação lateral da bola na unidade 4 correspondeu ao lançamento de bolas em toda a amplitude da mesa. Para o controle da freqüência de disparo de bolas também foram utilizados os ajustes do controle do equipamento que variam da unidade arbitrária de1 a 9. Essa unidade arbitrária foi manualmente convertida em bolas: min $^{-1}$ (convertida através da razão entre bolas disparadas e tempo na unidade arbitrária) O quique (pingo) da bola foi ajustado para ocorrer entre 50 a $60 \mathrm{~cm}$ à frente da rede. Esses ajustem foram efetuados para simular as respostas de um adversário em um jogo de tênis de mesa. Todos os procedimentos específicos foram realizados até a exaustão, que foi determinada instalada quando ocorreram quatro erros consecutivos nas rebatidas ou através da exaustão voluntária do participante.

\section{Teste de lactato mínimo específico (LACmin)}

Para a indução da hiperlactacidemia foi aplicado o teste Running Anaerobic Sprint Test (RAST) através da realização de seis corridas máximas na distância de 35 metros com intervalo de 10 segundos entre cada corrida. Após oito minutos de recuperação passiva foi iniciado um teste incremental na mesa utilizando o robô lançador de bolas (Robo Pro Plus, Tibhar, Alemanha) com freqüência inicial de disparo correspondente a aproximadamente 41 bolas min $^{-1}$ e aumento de aproximadamente 10 bolas min $^{-1}$ (aumento de uma unidade no ajuste do equipamento) a cada três minutos (estágio de exercício). Após cada estágio foi realizada uma pausa de 30 s para a coleta de sangue do lóbulo da orelha para análise da lactacidemia. Foram realizadas também coletas de sangue aos minutos 3, 5 e 7 após 0 teste de RAST e ao término do teste. A intensidade correspondente ao teste de lactato mínimo (LACmin) foi obtida através do ajuste polinomial de segunda ordem, sendo o LACmin correspondente à derivada zero (figura 1A). Esse mesmo procedimento foi aplicado após 24 horas para análise da reprodutibilidade do teste de lactato mínimo específico 


\section{Teste de potência crítica adaptado para o tênis de mesa (frequência crítica - fcrit)}

O teste de potência crítica foi adaptado para o tênis de mesa, e como o controle da intensidade foi realizado através da freqüência de disparo de bola, esse procedimento foi denominado freqüência crítica (fcrit). Os mesatenistas realizaram de três a quatro séries de exercícios em diferentes freqüências $(f)$ de disparo de bolas, sendo estas correspondentes a aproximadamente 52, 62, 72 e 77 bolas min $^{-1}$. As séries foram executadas até a exaustão e o tempo total de exercício (Tlim) em cada série foi registrado para a determinação na freqüência crítica (fcrit). As séries de exercício foram aplicadas de modo aleatório com intervalo mínimo de duas horas, mas realizadas apenas duas séries por dia. A intensidade correspondente à fcrit foi determinada através da relação linear entre freqüência de lançamentos de bolas ( $f$ ) pelo inverso do Tlim (1/Tlim), com a fcrit correspondente ao coeficiente linear da reta de regressão (intercepto-y) ${ }^{(7-9)}$ (figura 1B).

\section{Teste incremental específico para determinação do limiar anaeróbio}

Para a determinação do limiar anaeróbio em procedimento específico foi aplicado um teste incremental com intensidade inicial de 41 bolas: min $^{-1}$ e incremento de aproximadamente 10 bolas min $^{-1}$ a cada estágio de três minutos de exercício. Foram realizadas coletas de sangue após cada estágio (30 segundos) e também aos 3, 5 e 7 minutos após o término do teste. Através do comportamento da lactacidemia pela intensidade de esforço foi determinada a intensidade de limiar anaeróbio por dois métodos: 1) pela concentração fixa de 3,5 mM (LAn $\left.{ }_{\text {esp } 3,5}\right)$; e 2) método de regressão linear bissegmentada $\left(L A n_{B}\right)$ (figura $\left.1 C\right)$.

\section{DETERMINAÇÃO DO LIMIAR ANAERÓBIO EM CORRIDA}

\section{Determinação do limiar anaeróbio em corrida em esteira rolante $\left(\mathrm{LAn}_{\text {est }}\right)$}

O teste foi realizado em corrida em esteira rolante (ProAction BHfitness, Explorer, Espanha) com velocidade inicial correspondente a $7 \mathrm{~km} \cdot \mathrm{h}^{-1}$ e aumento de $1,5 \mathrm{~km} \cdot \mathrm{h}^{-1}$ a cada estágio de três minutos. A inclinação da esteira foi mantida constante a $1 \%$ como sugerido por Jones \& Doust ${ }^{(11)}$. Foram coletadas amostras de sangue ao final de cada estágio (30s de pausa) e também aos 3, 5 e 7 minutos após o final do teste. A intensidade de limiar anaeróbio foi correspondente à concentração fixa de lactato de $3,5 \mathrm{mM}^{(12)}$.

\section{Análise sanguínea}

Para a análise da lactacidemia foram coletadas $25 \mu \mathrm{L}$ (microlitros) de sangue do lóbulo da orelha do participante. As amostras foram analisadas em lactímetro eletroquímico (YSI 1500 Sport, Ohio, EUA) e a concentração de lactato expressa em mM.

\section{Análise estatística}

Inicialmente foi analisada a normalidade das variáveis através do teste de Shapiro-Wilk e todas as variáveis foram consideradas normais. Para análise da reprodutibilidade do teste de lactato mínimo em protocolo específico foram utilizados o teste de Bland-Altman ${ }^{(13)}$ e o teste de correlação produto-momento. $\mathrm{O}$ teste de análise de variância one way com teste post hoc de Newman-Keuls (caso necessário) foi utilizado para análise da igualdade entre as intensidades de lactato mínimo específico teste $\left(\mathrm{LACmin}{ }_{1}\right)$ e reteste $\left(\mathrm{LACmin} \mathrm{n}_{2}\right)$, freqüência crítica (fcrit), $L A n_{\text {esp } 3,5}$ e $L A n_{B \mid}$ nos testes específicos. Todos os procedimentos específicos determinados foram correlacionados pelo teste de correlação produto-momento e também com o limiar anaeróbio determinado em corrida. Em todos os casos foram utilizados níveis de significância de 5\%. Os resultados são expressos em média \pm desvio-padrão.

\section{RESULTADOS}

As intensidades de LACmin obtidas em LACmin ${ }_{1}$ e LACMin ${ }_{2}$ foram correspondentes a 59,40 \pm 4,06 bolas min $^{-1}$ e 61,56 $\pm 8,97$ bolas: $\mathrm{min}^{-1}$, respectivamente, não sendo diferentes estatisticamente e apresentando significativa correlação $(r=0,61)$. As concentrações de lactato nessas intensidades foram de 4,56 $\pm 1,26 \mathrm{mM}$ e 3,63 $\pm 1,26 \mathrm{mM}$ (respectivamente), e os coeficientes de determinação do ajuste polinomial correspondentes a $0,89 \pm 0,08$ e 0,89 $\pm 0,07$. A figura 2 apresenta a análise de gráfica de Bland-Altman ${ }^{(13)}$ entre as intensidades de LACMin ${ }_{1}$ e LACMin ${ }_{2}$. Foi verificada moderada concordância entre os resultados com média da diferença correspondente a $-2,7 \%$ e os limites superior e inferior correspondentes a $21,7 \%$ e $-27,2 \%$, respectivamente.

Os Tlim obtidos no teste de potência crítica adaptado para o tênis de mesa e denominado freqüência crítica (fcrit) nas intensidades cor-

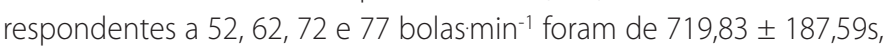
$377,82 \pm 150,22 \mathrm{~s}, 153,60 \pm 93,06 \mathrm{~s}, 73,71 \pm 29,48 \mathrm{~s}$, respectivamente. Foi obtido ótimo coeficiente de determinação da regressão linear para o tênis de mesa, correspondente a 0,86 $\pm 0,07$, e a intensidade de fcrit correspondente a $56,50 \pm 6,84$ bolas $\mathrm{min}^{-1}$. No teste incremental específico foram determinados o limiar anaeróbio através de dois procedi-
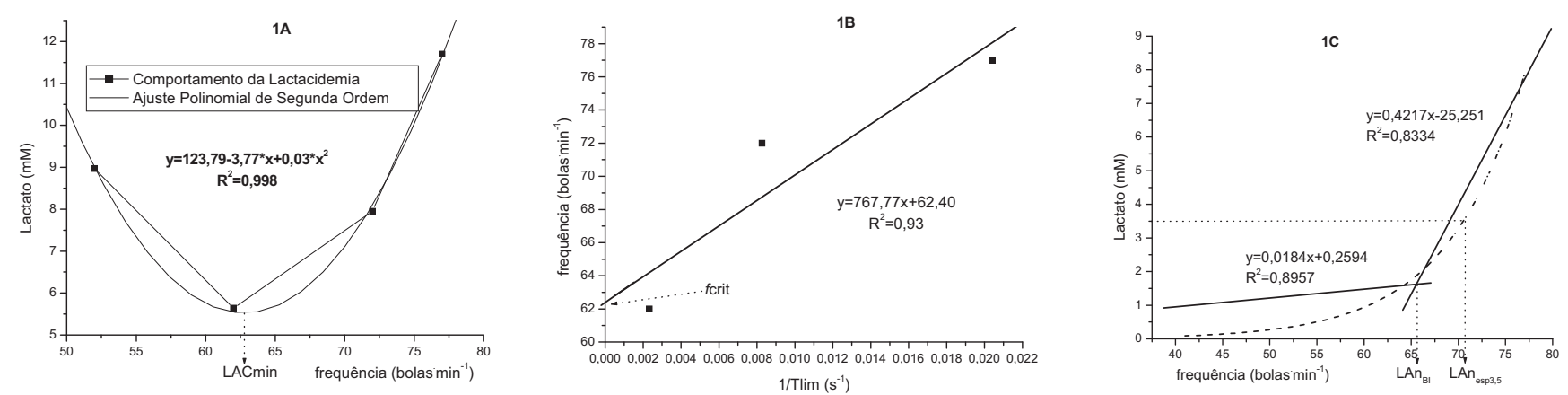

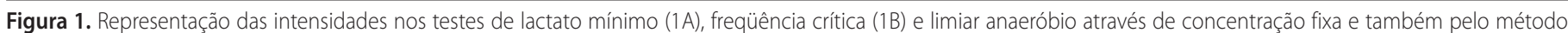
de regressão bissegmentada (1C) em testes específicos para o tênis de mesa utilizando robô lançador de bolas 


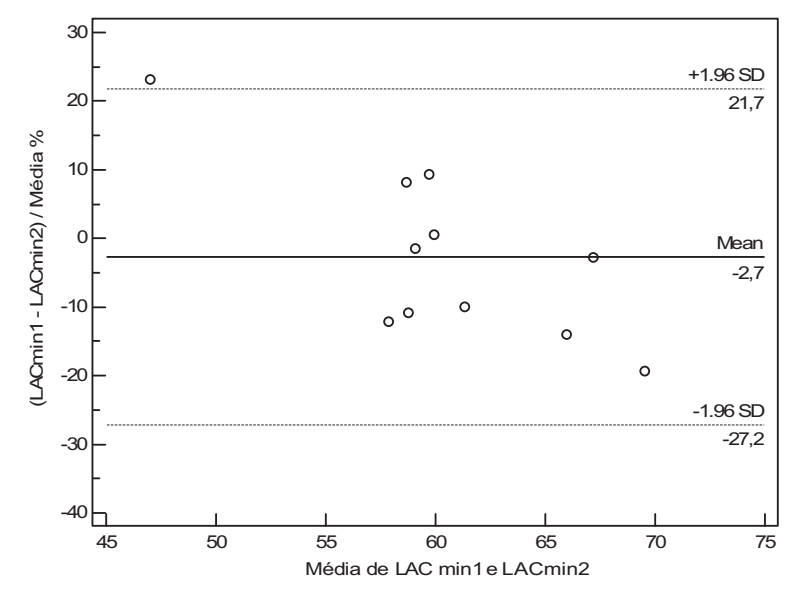

Figura 2. Análise gráfica de Bland-Altman (1986) entre as intensidades correspondentes aos testes de lactato mínimo específicos para o tênis de mesa em teste ( $\left(\mathrm{ACmin} \mathrm{n}_{1}\right)$ e reteste $(\mathrm{LACmin})$

mentos: o LAn $n_{\text {esp3,5 }}$ correspondeu a 66,74 $\pm 5,03$ bolas min $^{-1}$ e o $L_{A A n_{B l}}$

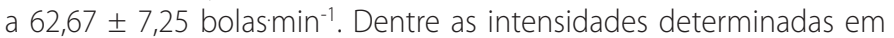
procedimentos específicos, apenas foi verificada diferença significativa entre as intensidades de $L A n_{\text {esp } 3,5}$ e fcrit. Foram encontradas correlações significativas entre LACmin e LACmin $_{2}(r=0,61)$, LACmin 1 e fcrit $(0,61), L A C \operatorname{Lin}_{2}$ e fcrit $(0,69), L A n_{\text {esp3,5 }}$ e LAn $n_{B I}(0,70)$. O limiar anaeróbio obtido em ergômetro convencional (esteira rolante) correspondeu a $9,11 \pm 1,94 \mathrm{~km} \cdot \mathrm{h}^{-1}$ e apresentou correlação significativa apenas com a intensidade de fcrit $(0,73)$.

\section{DISCUSSÃO}

Os principais achados do estudo foram a reprodutibilidade do teste de lactato mínimo adaptado para o tênis de mesa e a correlação do LACmin com a intensidade de freqüência crítica.

O teste de lactato mínimo específico para o tênis de mesa (LACmin) mostrou ser reprodutível por não ocorrer diferença entre as aplicações de teste $\left(\mathrm{LACmin} \mathrm{n}_{1}\right)$ e reteste $\left(\mathrm{LACmin} \mathrm{C}_{2}\right)$, apresentar significativa correlação entre essas intensidades $(r=0,61)$ e moderada concordância obtida através do teste de análise gráfica de Bland-Altman ${ }^{(13)}$. A média da diferença obtida no teste de Bland-Altman ${ }^{(13)}$ foi próxima a zero e apresenta apenas um ponto fora dos limites de aceitação (outliers), mas as variações dos limites de aceitação foram de aproximadamente $25 \%$.

O teste de lactato mínimo determina a aptidão aeróbia através do ponto de equilíbrio entre produção e remoção de lactato de maneira individual(14) e tem mostrado ser boa ferramenta na predição da intensidade referente à máxima fase estável de lactato(15,16). Esse teste possibilita em um único procedimento verificar a aptidão aeróbia e anaeróbia do avaliado; isso, do ponto de vista prático, torna-se muito interessante. Mas esse procedimento ainda não foi adaptado para as modalidades esportivas que utilizam raquetes. Para esses esportes, como o tênis, o badminton, o squash e o tênis de mesa, geralmente são encontrados estudos que utilizaram procedimentos mais clássicos, como o limiar anaeróbio(2,3), o limiar ventilatório(5), o consumo máximo de oxigênio(2,6) e a PWC90(4). No tênis de mesa, apenas o modelo de potência crítica adaptado (freqüência crítica) foi investigado e validado ${ }^{(7-9)}$; ainda é um esporte que apresenta muito poucos estudos. No estudo realizado por Zagatto \& Gobatto ${ }^{(7)}$ foi verificada a reprodutibilidade da fcrit aplicada para o tênis de mesa, na qual posteriormente foi validado verificando estabilização na concentração de lactato na intensidade de $100 \%$ da fcrit em exercício de longa duração e também a correlação da fcrit com limiar anaeróbio determinado pelo método bissegmentado $(r=0,71)^{(8)}$. Zagatto \& Gobatto ${ }^{(9)}$ também verificaram que na intensidade correspondente a fcrit não ocorreu restauração da reserva energética anaeróbia (CTA), e que o Tlim real na intensidade de 150\% da fcrit em condição de depleção anaeróbia (metade da CTA utilizada previamente) foi o mesmo que o Tlim teórico, corroborando a validade do procedimento. Zagatto et al. ${ }^{(10)}$, ainda investigando o modelo de potência crítica aplicado ao tênis de mesa, relataram que o parâmetro anaeróbio desse modelo denominado capacidade de trabalho anaeróbio (CTA) não parece ser um bom índice da aptidão anaeróbia no tênis de mesa, sendo indicado apenas o componente aeróbio do teste de fcrit como um bom procedimento de avaliação. Como apenas esse procedimento foi validado para o tênis de mesa e o componente aeróbio desse modelo já é bem validado em outros eventos esportivos ${ }^{(17-19)}$, utilizamos esse teste como procedimento padrão, embora seja um teste não invasivo.

Nos testes aplicados em protocolo específico foi obtida diferença significativa apenas para a intensidade de freqüência crítica e a intensidade de limiar anaeróbio determinado pela concentração fixa de lactato de 3,5mM ( $\left(\mathrm{An}_{\mathrm{esp} 3,5}\right)$. Embora a utilização da concentração fixa possa ser um procedimento prático e muito aplicado para avaliar o componente aeróbio, essa proposta foi inicialmente indicada para a corrida ${ }^{(12)}$ e, posteriormente natação ${ }^{(20)}$, pois esses esportes apresentam concentrações de lactato na MFEL próximos a esse valor. Mas já foi demonstrado que a concentração de lactato na MFEL é dependente do evento motor realizado(21) e esse valor de lactacidemia pode não ser o mesmo para o tênis de mesa; desse modo, a utilização da concentração fixa de lactato para determinação LAn deve ser utilizada com cautela. Embora a concentração de lactato no LACmin 2 foi equivalente a $3,63 \pm 1,26 \mathrm{mM}$ e próximo ao valor de $3,5 \mathrm{mM}$, a resposta da [LAC] no teste de lactato mínimo é dependente da intensidade inicial, número de estágios até atingir a intensidade de LACmin e também da [LAC] após a indução da hiperlactacidemia; desse modo, não pode ser utilizada como um bom parâmetro. A intensidade de LACmin 1 e a de LACmin foram significativamente correlacionadas com a fcrit ( $r=0,61$ e $r=0,69$, respectivamente), fortalecendo a aplicação do teste de LACmin como um bom procedimento de avaliação no tênis de mesa. Porém, as intensidade de LACmin não apresentaram correlações significativas com as intensidades de $L A n_{B I}$ e $L A n_{\text {esp } 3,5}$, resultado também verificado com a fcrit. Além dos problemas encontrados na utilização da concentração fixa para determinação do LAn mencionados anteriormente, talvez o fato de terem sido utilizados poucos estágios de exercício (cinco em média) para a determinação do limiar anaeróbio através dessa regressão bissegmentado pode ter influenciado diretamente a obtenção de duas retas seguras, podendo resultar em possíveis erros na determinação do LAn. A utilização de um número maior de pontos talvez possa melhorar os ajustes lineares e determinar o limiar anaeróbio com maior precisão no tênis de mesa. Contudo, a aplicação do teste incremental para determinação do limiar anaeróbio no tênis de mesa ainda precisa ser mais bem investigada; com os resultados obtidos neste estudo, parece não ser possível sua aplicação.

A elaboração de um procedimento específico para o tênis de mesa é muito difícil, pois muitas variáveis são utilizadas para a obten- 
ção da ótima performance no jogo. Nesse estudo utilizamos apenas ataques de forehand, mas não podemos deixar de mencionar que nesse esporte também existem outros tipos de golpes, como chopes, bloqueios, loop e smash, que são importantes para o rendimento no jogo ${ }^{(22)}$, mas de incorporação difícil em um procedimento de avaliação.

Quando analisada a relação entre os procedimentos específicos com o teste de LAn em esteira, foi verificada apenas correlação do $L A n_{\text {est }}$ com fcrit $(r=0,73)$, sendo esse um fraco indício para a indicação da utilização da esteira rolante como procedimento para avaliar a aptidão aeróbia no tênis de mesa. Diversos estudos têm demonstrado a necessidade do uso de testes específicos para a avaliação da aptidão aeróbia, inclusive em esportes com raquetes ${ }^{(1-3,5)}$. Zagatto et al. ${ }^{(23)}$ compararam o resultado da intensidade de fcrit determinada em ergômetro específico para o tênis de mesa com o LACmin obtido em ergômetro de braço e cicloergômetro, e não verificaram correlações significativas. Resultado semelhante foi verificado por Smekal et al.(1) em relação ao consumo máximo de oxigênio obtido em testes realizados em esteira e em ergômetro específico para o tênis.
Desse modo, podemos concluir que o teste de lactato mínimo específico para o tênis de mesa é reprodutível, que esse procedimento e o teste de freqüência crítica podem ser aplicados para avaliar a transição no tênis de mesa, e que o teste incremental para determinação do LAn em procedimento específico através de concentração fixa de lactato e também pelo modelo de regressão linear bissegmentada parece não ser adequado, mas deve ser mais bem investigado. Ainda, a esteira rolante para determinação da aptidão aeróbia em mesatenistas deve ser utilizada com cautela agregada a um procedimento específico, e não como único procedimento de avaliação.

\section{AGRADECIMENTOS}

Apoio Financeiro da Fundect - Fundação de Apoio ao Desenvolvimento do Ensino, Ciência e Tecnologia do Estado de Mato Grosso do Sul (Processos 41/100.111/2006 e 41/100.187/2006), UFMS e CAPES.

Todos os autores declararam não haver qualquer potencial conflito de interesses referente a este artigo.

\section{REFERÊNCIAS BIBLIOGRÁFICAS}

1. Smekal G, Pokan R, Von Duvillard SP, Baron R, Tschan H, Bachl N. Comparison of laboratory and "oncourt" endurance testing in tennis. Int J Sports Med 2000;21:242-9.

2. Girard O, Chevalier R, Leveque F, Micallef JP, Millet GP. Specific incremental field test for aerobic fitness in tennis. Brit J Sports Med 2006:40:791-6.

3. Chin MK, Wong AS, So RC, Siu OT, Steininger K, Lo DT. Sport specific fitness testing of elite badminton players. Brit J Sports Med 1995;29:153-7.

4. Wonisch M, Hofmann P, Schwaberger G, Von Duvillard SP, Klein W. Validation of a field test for the non-invasive determination of badminton specific aerobic performance. Brit J Sports Med 2003;37:115-8.

5. Girard O, Sciberras P, Habrard M, Hot P, Chevalier R, Millet GP. Specific incremental test in elite squash players. Brit J Sports Med 2005;39:921-6.

6. Smekal G, Von Duvillard SP, Rihacek C, Pokan R, Hofmann P, Baron R, et al. A physiological profile of tennis match play. Med Sci Sports Exerc 2001;33:999-1005

7. Zagatto AM, Gobatto, CA. Determinação de um modelo de avaliação aeróbia no tênis de mesa em protocolo específico utilizando robô. Table Tennis Player 2002;15:10-1.

8. Zagatto AM. Determinação da freqüência crítica e da capacidade de trabalho anaeróbio no tênis de mesa em protocolo específico utilizando robô. Dissertação (Mestrado em Ciência da Motricidade). Universidade Estadual Paulista (UNESP). Rio Claro, 2004

9. Zagatto AM, Gobatto CA. Validação do modelo de freqüência crítica em protocolo específico através de método indireto, para o tênis de mesa. Lecturas Educación Física y Deportes 2007;110:1-7.

10. Zagatto AM, Papoti M, Gobatto CA. Anaerobic capacity may not be determined by critical power model in elite table tennis players. J Sports Sci Med 2008;7:54-59.

11. Jones AM, Doust JH. A $1 \%$ treadmill grade most accurately reflects the energetic cost of outdoor running. J Sports Sci 1996;14:321-7.

12. Heck H, Mader A, Hess G, Mücke S, Muller R, Hollmann W. Justification of the 4-mmol// lactate threshold. Int J Sports Med 1985;6:117-30.
13. Bland JM, Altman DG. Statistical methods for assessing agreement between two methods of clinical measurement. Lancet 1986;8:307-10.

14. Tegtbur U, Busse MW, Braumann KM. Estimation of an individual equilibrium between lactate production and catabolism during exercise. Med Sci Sports Exerc 1993;25:620-7

15. Bacon L, Kern M. Evaluating a test protocol for predicting maximum lactate steady state. J Sports Med Phys Fitness 1999;39:300-8.

16. Jones AM, Doust JH. The validity of the lactate minimum test for determination of the maximal lactate steady Med Sci Sports Exerc 1998;30:1304-13.

17. Wakayoshi K, Yoshida T, Udo M, Harada T, Moritani T, Mutoh Y, et al. Does critical swimming velocity represent exercise intensity at maximal lactate steady state? Eur J Appl Physiol 1993;66:90-5.

18. Smith CGM, Jones AM. The relationship between critical velocity, maximal lactate steady-state velocity and turn point velocity in runners. Eur J Appl Physiol 2001;85:19-26.

19. Papoti M, Zagatto AM, Mendes OC, Gobatto CA. Utilização de métodos invasivo e não invasivo na predição das performances aeróbia e anaeróbia em nadadores de nível nacional. Revista Portuguesa de Ciências do Desporto 2005;5:7-14.

20. Pereira RR, Papoti MP, Zagatto AM, Gobatto CA. Validação de dois protocolos para determinação do limiar anaeróbio em natação. Motriz 2002;8:63-8.

21. Beneke R, Von Duvillard SP. Determination of maximal lactate steady state response in selected sports events. Med Sci Sports Exerc 1996;28:241-6.

22. Toriola AL, Toriola OM. Validity of specific motor skills in predicting table-tennis performance in novice players. Perceptual and Motor Skills 2004;98:584-6.

23. Zagatto AM, Papoti M, Caputo F, Mendes OC, Denadai BS, Baldissera V, et al. Comparação entre a utilização de saliva e sangue para determinação do lactato mínimo em cicloergômetro e ergômetro de braço em mesa-tenistas. Rev Bras Med Esporte 2004;10:475-80. 